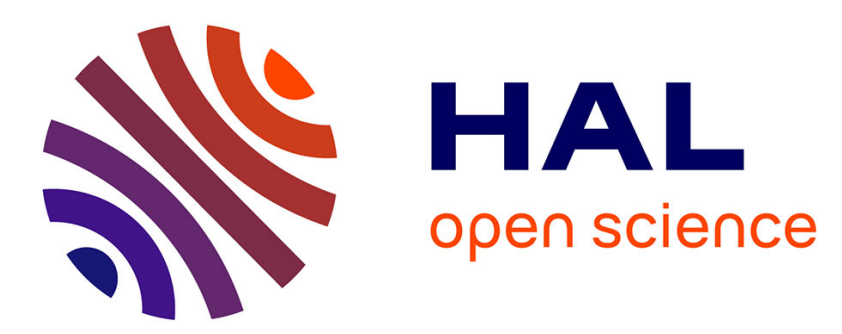

\title{
Saturation dans les expériences de double résonance hertzien-hertzien
}

P. Glorieux, J. Legrand, B. Macke, J. Messelyn

\section{To cite this version:}

P. Glorieux, J. Legrand, B. Macke, J. Messelyn. Saturation dans les expériences de double résonance hertzien-hertzien. Revue de Physique Appliquée, 1971, 6 (3), pp.389-400. 10.1051/rphysap:0197100603038900 . jpa-00243561

\section{HAL Id: jpa-00243561 https://hal.science/jpa-00243561}

Submitted on 1 Jan 1971

HAL is a multi-disciplinary open access archive for the deposit and dissemination of scientific research documents, whether they are published or not. The documents may come from teaching and research institutions in France or abroad, or from public or private research centers.
L'archive ouverte pluridisciplinaire HAL, est destinée au dépôt et à la diffusion de documents scientifiques de niveau recherche, publiés ou non, émanant des établissements d'enseignement et de recherche français ou étrangers, des laboratoires publics ou privés. 


\title{
SATURATION DANS LES EXPÉRIENCES DE DOUBLE RÉSONANCE HERTZIEN-HERTZIEN (*)
}

\author{
P. GLORIEUX, J. LEGRAND, B. MACKE et J. MESSELYN
}

Laboratoire de Spectroscopie Hertzienne, Equipe associée au C. N. R. S., Université de Lille I

(Reçu le 17 février 1971, révisé le 26 avril 1971)

\begin{abstract}
Résumé. - Les auteurs ont mis en évidence des phénomènes de saturation dus à la puissance de sonde dans les expériences de double résonance hertzien-hertzien. L'accord entre les résultats expérimentaux et une théorie basée sur le formalisme de l'hamiltonien habillé est tout à fait satisfaisant.

Abstract. - The authors exhibit signal saturation phenomena in microwave double resonance experiments. The agieement between theoretical calculations by means of the dressed hamiltonian formalism and experimental results is fairly good.
\end{abstract}

Introduction. - Les études de double résonance sur deux transitions de rotation d'un gaz à faible pression ont fait l'objet de nombreux travaux, tant théoriques qu'expérimentaux [1]. Rappelons rapidement le principe de ces expériences sur l'exemple particulier que nous avons étudié [2], [3]. Soient 1,2 et 3 des niveaux d'énergie de rotation de la molécule $\mathrm{SO}_{2}$ d'anhydride sulfureux [4] (respectivement niveaux $6_{06}$, $6_{15}$ et $5_{24}$ dans la notation de Mulliken).

Les transitions $1 \rightarrow 2$ et $2 \rightarrow 3$ sont permises et sont définies par leurs fréquences $v_{0}^{\prime}$ et $v_{0}$ respectivement $\left(v_{0}^{\prime}=68972 \mathrm{MHz} ; v_{0}=23414 \mathrm{MHz}\right)$; la transition $1 \rightarrow 3$ est interdite (approximation dipolaire électrique). Les deux transitions $1 \rightarrow 2$ et $2 \rightarrow 3$ ayant un niveau commun, on conçoit qu'une puissance envoyée à une fréquence $v^{\prime}$ voisine de $v_{0}^{\prime}$ sur le gaz modifie son comportement aux fréquences $v$ voisines de $v_{0}$. Le gaz est donc soumis à une double irradiation à la fréquence $v^{\prime}$ (pompe) d'une part, et à la fréquence $v$ (sonde) d'autre part. La détection du signal d'absorption s'effectue à la seule fréquence $v$ grâce à un dispositif de filtres convenable. Toutes les études de ce type ont porté exclusivement sur l'absorption dans le cas où la puissance de sonde est très petite devant la puissance de pompe et, implicitement, en supposant les effets de saturation de sonde négligeables. Dans une publication antérieure [5], nous avons montré que l'absorption s'accompagnait d'une dispersion, c'est-à-dire une variation d'indice, et nous avons proposé une technique de calcul [6], [7] du terme de dispersion basé sur le formalisme de l'opérateur d'évolution. Nous publierons

(*) Ce travail constitue le résumé d'une partie de la thèse de Doctorat d'Etat de Sciences Physiques soutenue par B. Macke le 24/10/70 devant la Faculté des Sciences de Lille, C. N. R. S., $\mathrm{n}^{\circ} \mathrm{AO} 5221$. prochainement des résultats plus complets sur ce sujet. Par ailleurs, les études expérimentales de la dispersion nous ont amenés à travailler à des pressions assez basses ( 2 millitorrs environ) pour lesquelles la saturation de sonde est inévitable. Pour mieux connaître ces effets et leur incidence sur les figures d'absorption et de dispersion, nous avons entrepris leur étude expérimentale essentiellement dans le cas de l'absorption. Du point de vue théorique, les traitements habituellement proposés ne permettent pas de prendre en compte les effets de saturation de sonde. Récemment, Di Giacomo et Santucci [8] ont proposé d'appliquer aux problèmes de double résonance le formalisme de l'hamiltonien habillé et ont montré dans un cas particulier, et de façon approchée, l'existence d'effets de saturation de sonde. En reprenant leur étude dans un cadre plus général [9] c'est-à-dire en faisant jouer des rôles parfaitement symétriques aux signaux de pompe et de sonde, nous avons développé une théorie bien adaptée à l'interprétation de nos résultats expérimentaux.

Après avoir indiqué le dispositif expérimental employé, nous commenterons quelques enregistrements caractéristiques. Nous interpréterons ensuite ces effets à l'aide d'une théorie basée sur le formalisme de l'hamiltonien habillé et nous montrerons enfin que les écarts entre les résultats théoriques et expérimentaux peuvent être attribués à l'inhomogénéité de la puissance de pompe et aux effets de dégénérescence $m$.

1. Dispositif expérimental. - Dans la plupart des spectromètres récents destinés aux études de double résonance, on satisfait aux exigences de sensibilité en réalisant une modulation de l'absorption et une démodulation synchrone du signal détecté à la fréquence de sonde. L'utilisation de balayage lent des fréquences 
impose dans ce cas l'utilisation de dispositifs de stabilisation des fréquences de pompe et de sonde.

Dans le spectromètre décrit par Messelyn [3], la stabilisation des fréquences est assurée par des servomécanismes d'asservissement en phase. On module l'absorption grâce à une modulation par tout ou rien de la puissance de pompe. Nous avons également retenu ces procédés mais nous avons étendu nos possibilités expérimentales grâce à des techniques de traitement des signaux par échantillonnage et par détection superhétérodyne.

Nous décrirons les solutions pratiques apportées aux différents problèmes de réalisation posés par le montage hyperfréquence, l'asservissement en phase des oscillateurs, le balayage de leur fréquence, le traitement du signal et les mesures de fréquences. Nous indiquerons enfin les performances du spectromètre ainsi réalisé.

I. 1 MONTAgE HYPERFRÉQUENCE. - La cellule d'absorption est réalisée en guide $3 \mathrm{~cm}$ (RG $52 \mathrm{U}$ ) surdimensionné pour les fréquences de pompe et de sonde. Elle est constituée de quatre sections de $3 \mathrm{~m}$ assemblées à l'aide de coudes $180^{\circ}$. Nous avons choisi des coudes $\mathrm{E}$ avec lesquels les réflexions sont moins sensibles. Les brides de ces coudes ont une gorge recevant un joint torique pour assurer l'étanchéité des raccords. L'étanchéité à l'entrée et à la sortie de la cellule est réalisée par deux fenêtres en mica d'épaisseur $50 \mu$ serrées entre deux brides avec joints intermédiaires en indium. Le pompage s'effectue à une extrémité de la cellule par un groupe constitué d'une pompe secondaire à diffusion d'huile et d'une pompe primaire à palettes. La communication avec la cellule est assurée par un orifice de $2 \mathrm{~cm}^{2}$ de section réalisé en abattant la paroi du guide sur son petit côté.

Ceci permet une grande vitesse de pompage et n'affecte pas trop la courbe de transmission de notre cellule. Le gaz est introduit à l'autre extrémité de la cellule et son admission est contrôlée par un robinet de fuite à aiguille de faible conicité (Hoke $1335 \mathrm{M} 4$ y). La plupart des expériences sont réalisées en régime d'écoulement, le gaz étant introduit et pompé en permanence. Ceci permet de garantir une bonne pureté du gaz aux pressions les plus basses utilisées $(0,5$ millitorr). La pression est ajustée en réglant le débit du robinet à fuite. Ce réglage est rendu plus facile en refroidissant la réserve d'anhydride sulfureux par un mélange réfrigérant carboglace-acétone, ce qui ramène sa pression de vapeur saturante à 200 millitorrs. La pression est mesurée au milieu de la cellule à l'aide d'une jauge de Mc Leod et repérée par une jauge de Pirani.

La figure 1 donne un schéma d'ensemble du spectromètre. L'oscillateur de sonde est un klystron $1,25 \mathrm{~cm}$ (E. M. I. R 9675), le pompage $5 \mathrm{~mm}$ étant effectué par un carcinotron (C. S. F. COE 40 B). L'introduction des deux puissances s'effectue par des coupleurs directifs $3 \mathrm{~dB}$ en guide RG $53 \mathrm{U}$. Le signal de pompe introduit par la voie directe est partiellement piégé par la

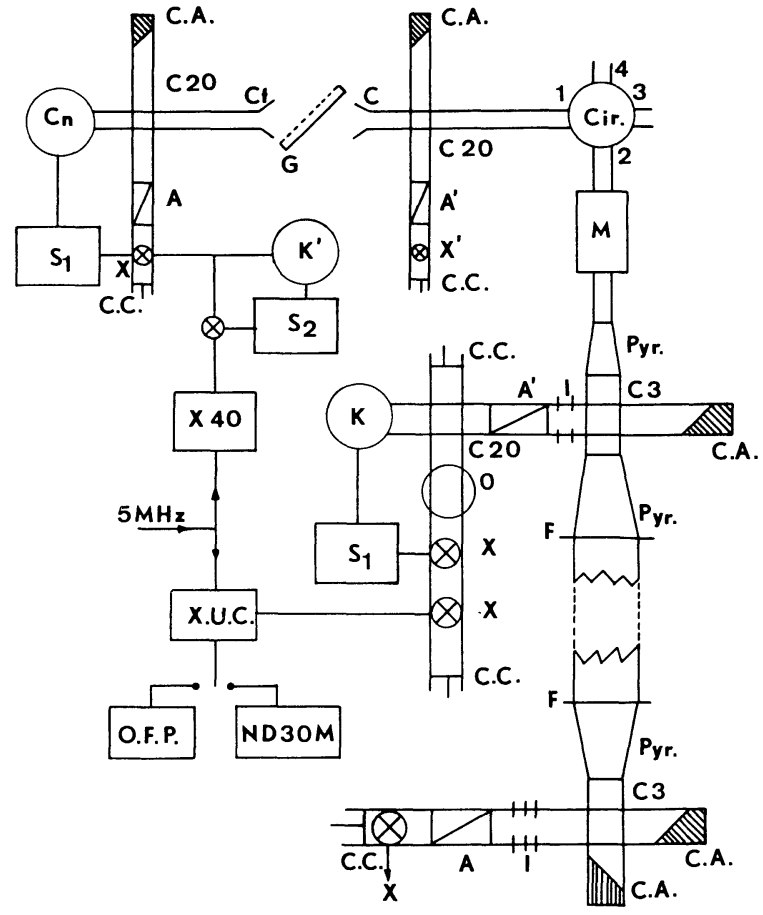

FIg. 1. - Spectromètre à ondes progressives pour étude des phénomènes de saturation d'absorption. A : Atténuateur; $\mathrm{A}^{\prime}$ : Atténuateur calibré; $\mathrm{C} 3$ : Coupleur directif $3 \mathrm{~dB} ; \mathrm{C} 20$ : Coupleur directif $20 \mathrm{~dB}$; C. A. : Charge Adaptée ; C. C. : Piston de Court-Circuit ; Cir. : Circulateur commutable; $\mathrm{Cn}$ : Carcinotron $4 \mathrm{~mm} \mathrm{C.} \mathrm{S.} \mathrm{F.} \mathrm{COE} \mathrm{40B} \mathrm{;} \mathrm{Ct} \mathrm{:} \mathrm{Cornet} \mathrm{hyperfréquence} 5 \mathrm{~mm}$; $\mathrm{G}$ : Grille polarisante Philips ; I : Iris résonnant à la fréquence de sonde ; K : Klystron de sonde E. M. I. ; K' : Klystron férisol ; M : Modulateur C. S. F. 50-75 Mod.; NDM 30 : Synthétiseur de fréquence $300 \mathrm{~Hz} / 30 \mathrm{MHz}$; O : Ondemètre ; O. F. P. : Oscillateur à Fréquence Programmable ; Pyr. : Transition Pyramidale ; $\mathrm{S}_{1}$ : Synchriminateur de phase Schomanli FDS $30 ; S_{2}$ : Synchriminateur de phase Dymec; $\mathrm{X}, \mathrm{X}^{\prime}$ : Cristaux détecteurs, mélangeurs ou multiplicateurs; $X$ 40: Multiplicateur $5 \mathrm{MHz} \rightarrow 200 \mathrm{MHz}$; X. U. C. : Synthétiseur de fréquence $470-1000 \mathrm{MHz} ; \mathrm{F}$ : Fenêtre de mica.

grille de couplage mais ceci n'a guère d'importance dans les études de saturation de sonde. Pour maîtriser la puissance importante issue de carcinotron $(11 \mathrm{~W}$ au maximum) et découpler celui-ci de la charge, nous avons réalisé une propagation quasi optique. La puissance est aisément contrôlée à l'aide de la grille à fils parallèles placée entre les deux cornets. Le cornet de réception est suivi d'un coupleur $20 \mathrm{~dB}$. L'atténuateur calibré (Hitachi M 1513), placé dans la voie latérale permet, en maintenant le signal du détecteur $\mathrm{X}^{\prime}$ à un niveau constant, d'obtenir un repère précis de la puissance de pompe (donnée relative).

Nous disposons de la possibilité de moduler par tout ou rien la puissance de pompe. Pour éviter que cette modulation ne perturbe gravement le fonctionnement de la source hyperfréquence, nous avons abandonné le modulateur précédemment utilisé pour lui substituer un circulateur commutable.

En l'absence d'excitation, ce circulateur fonctionne selon la séquence $1,2,3,4$ et, en lui appliquant une excitation convenable, il fonctionne selon la séquence 
inverse 4, 3, 2, 1. En utilisant la transmission de 1 vers 2 , en plaçant des charges adaptées (cornets) en 3 et 4 , on réalise la commutation cherchée sans variation sensible de la puissance réfléchie vers la source.

La puissance de sonde est introduite par la voie dérivée du coupleur $3 \mathrm{~dB}$. Elle est contrôlée par un atténuateur calibré ; celui-ci est protégé des remontées de la puissance de pompe par un iris résonnant à la fréquence de sonde (fenêtre de $1,00 \times 6,07 \mathrm{~mm}$ ) qui assure une atténuation de l'ordre de $20 \mathrm{~dB}$ à la fréquence de pompe. Un coupleur $20 \mathrm{~dB}$ prélève la partie de la puissance de sonde nécessaire au détecteur à deux cristaux de la chaîne d'asservissement en phase du klystron.

Le signal utilisé pour l'asservissement en phase du carcinotron est prélevé à l'aide d'un coupleur $20 \mathrm{~dB}$ avant le dispositif quasi optique. Le même cristal réalisant la multiplication et le mélange de fréquences, un atténuateur est nécessaire pour ajuster le niveau à l'optimum.

A l'extrémité de la cellule d'absorption, une charge adaptée dissipe la majeure partie de la puissance de pompe. La moitié de la puissance de sonde est prélevée à l'aide d'un deuxième coupleur directif, une série de trois iris résonnants identiques à celui déjà décrit assure une protection de $60 \mathrm{~dB}$ contre la puissance de pompe qui traverse la grille du coupleur. Les deux atténuateurs placés devant le détecteur permettent, quelle que soit la puissance de sonde utilisée, de maintenir le courant continu détecté à une valeur constante. Ceci permet d'une part d'assurer une sensibilité constante et d'autre part d'obtenir un signal alternatif détecté directement proportionnel à la puissance relative absorbée. Ce point est particulièrement important dans les études de saturation.

I. 2 ASSERVISSEMENTS EN PHASE DES OSCILlateURS DE POMPE ET DE SONDE. - Ils sont nécessaires en raison des stabilités et des précisions requises par l'étude entreprise.

Ils sont réalisés à l'aide de synchriminateurs Schomandl FDS 30 dont la fréquence intermédiaire $F_{i}$ est comprise dans la gamme 28-32 MHz. Suivant les expériences entreprises, on adopte une fréquence fixe de $30 \mathrm{MHz}$ obtenue par multiplication de la fréquence d'un étalon $5 \mathrm{MHz}$ General Radio $1115 \mathrm{~B}$ ou une fréquence variable fournie par un oscillateur à fréquence programmable (O.F. P.). L'étalon radiofréquence utilisé pour l'asservissement du klystron de sonde est un synthétiseur de fréquence XUC Rohde et Schwarz également piloté par l'étalon $5 \mathrm{MHz}$. Ce synthétiseur fournit des fréquences discrètes de $10 \mathrm{MHz}$ en $10 \mathrm{MHz}$ entre 470 et $1000 \mathrm{MHz}$. Les fréquences intermédiaires entre ces fréquences discrètes peuvent être obtenues grâce à un interpolateur 20-30 MHz. Suivant les cas, cet interpolateur est constitué par un synthétiseur «Basse Fréquence» Schomandl ND $30 \mathrm{M}$ ou un oscillateur à fréquence programmable. (Dans la suite du texte, nous désigne- rons les oscillateurs de ce type par leurs initiales O. F. P.)

L'étalon radiofréquence utilisé pour l'asservissement en phase du carcinotron de pompe est un klystron 2-4, $3 \mathrm{GHz}$ (Férisol o S 401) lui-même asservi en phase à l'aide du dispositif Dymec 2650 A. Cette première stabilisation utilise une radiofréquence de $200 \mathrm{MHz}$ obtenue par multiplication $(\times 40)$ de la fréquence de l'étalon $5 \mathrm{MHz}$ et une fréquence intermédiaire $f_{\mathrm{i}}$ qui est réalisée de la même manière que la fréquence intermédiaire $F_{\mathbf{i}}$.

I. 3 BALAYAge DES FRÉQUENCES. - Le balayage des fréquences de sonde ou de pompe est assuré par l'O.F. P. de la chaîne d'asservissement correspondante. Signalons les difficultés que nous avons rencontrées dans le balayage du carcinotron dans un large intervalle de fréquences; nous avons remédié à ces difficultés en utilisant la technique de préconduite [10] généralement citée à propos des asservissements en fréquence. On applique à chaque instant sur la ligne du carcinotron la tension nécessaire pour annuler la tension d'erreur du servomécanisme d'asservissement. On obtient approximativement ce résultat en appliquant à la ligne une tension proportionnelle à celle appliquée à l'oscillateur programmable. Lorsque le coefficient de proportionnalité est correctement ajusté, la seule tension de correction apportée par le synchriminateur de phase est due aux défauts de linéarité tension/fréquence. Pratiquement, en raison du fort courant de ligne du carcinotron, la tension de préconduite ne peut être appliquée directement. Elle est appliquée par l'intermédiaire d'un circuit passif à transistors qui est la reconduction de celui réalisé dans le synchriminateur et placé en série avec lui.

En raison des exigences de stabilité et de vitesse de balayage, les O.F.P. ont fait l'objet d'une étude particulière [11].

Les tensions de programme appliquées aux circuits de commande sont généralement fournies par un générateur de signaux triangulaires de période comprise entre une milliseconde et mille secondes (C. R. C. GB. 860). Les signaux triangulaires sont plus favorables pour un fonctionnement correct des chaînes d'asservissement en phase que les signaux en dents de scie. Le même générateur fournit éventuellement la tension de préconduite indispensable dans le cas de larges intervalles de fréquences balayés.

1.4 Traitement DU SIGNAL DÉTECTÉ. - Les expériences de double résonance se prêtent bien à la réalisation d'un spectromètre à modulation d'absorption.

On peut réaliser cette modulation en modulant par tout ou rien la puissance de pompe ou en balayant périodiquement la fréquence de pompe. A ces deux possibilités, correspondent deux traitements possibles du signal : la démodulation synchrone et l'échantillonnage.

Dans l'utilisation de la technique de démodulation synchrone, la puissance de pompe est hachée grâce au 
circulateur commutable T. R. G. V 162 commandé par son générateur Ferrite Driver T. R. G. 171. La fréquence de commutation est de $4,5 \mathrm{kHz}$. Le signal issu du cristal d'extrémité de cellule est appliqué à un ensemble de détection de phase constitué d'un préamplificateur apériodique à faible bruit et d'un détecteur synchrone P. A. R. 121. La référence nécessaire au détecteur synchrone (fonctionnement « externe ») est prélevée directement sur la bobine d'excitation du circulateur.

Après ce traitement, on obtient un signal continu proportionnel à la différence des absorptions de sonde en présence et en absence de pompage. Bien entendu, en balayant lentement la fréquence de pompe ou de sonde, l'autre fréquence étant fixe, on peut faire des études de figures d'absorption.

Généralement, nous avons utilisé une constante de temps de 100 à $300 \mathrm{~ms}$ et une durée de balayage de 50 à $200 \mathrm{~s}$. Les signaux sont enregistrés sur un enregistreur graphique Hewlett Packard 7035 AM. Une difficulté rencontrée dans cette étude est la réjection parfois insuffisante du circulateur commutable. Cette réjection est de $26 \mathrm{~dB}$ et, de ce fait, on observe un signal proportionnel à la différence des absorptions de sonde en présence d'une puissance de pompe $\mathbf{P}^{\prime}$ et en présence d'une puissance inférieure de $26 \mathrm{~dB}\left(\mathbf{P}^{\prime} / 400\right)$. Dans le cas de forts pompages, l'effet obtenu pour $\mathrm{P}^{\prime} / 400$ peut être notablement différent au voisinage des fréquences $v_{0}$ et $v_{0}^{\prime}$ de celui qui serait observé à puissance de pompe nulle. Ceci peut donc entraîner une difficulté d'interprétation de certains enregistrements. On peut y remédier partiellement en doublant le circulateur par un modulateur classique (C. S. F. 50-75 Mod) excité en synchronisme.

La technique d'échantillonnage ne présente pas de telles difficultés [12], [13], [14]. Elle consiste à balayer périodiquement l'une des fréquences $v^{\prime}$ ou $v$ et à intégrer la figure d'absorption sur un grand nombre de balayages. Pratiquement, le balayage de la fréquence de sonde $v$ n'est guère utilisable car le signal d'absorption est entièrement masqué par les variations de transmission de la cellule qui dépend de la fréquence. En revanche, en modulant la fréquence de pompe $v^{\prime}$ pour une fréquence $v$ de sonde fixée, on ne module que l'absorption et la technique de traitement des signaux périodiques est bien adaptée. Bien entendu, l'échantillonneur fournit un signal de valeur moyenne nulle mais on sait que, lorsque la fréquente $v^{\prime}$ de pompe est suffisamment éloignée de la fréquence $v_{0}^{\prime}$ de la transition, l'absorption à la fréquence $v$ est la même que celle qui est observée en absence de pompage. Pratiquement, nous utilisons le Waveform Eductor TDH-9 P. A. R. qui est un échantillonneur analogique à 100 canaux. La partie du signal étudié est décomposée en cent intervalles de temps auxquels correspondent un échantillon. Ces échantillons étant toujours positionnés de la même manière par rapport au début du cycle de balayage, on réalise une intégration sur un très grand nombre $n$ de cycles pour obtenir une amélioration du rapport signal sur bruit dont la valeur théorique est $\sqrt{n}$. Le choix de la période de balayage résulte d'un compromis ; le rapport signal/bruit est d'autant meilleur pour une constante d'intégration donnée, que cette période est plus petite.

En revanche, aux grandes vitesses de balayage, on peut craindre des anomalies dans le fonctionnement de la chaîne d'asservissement en phase de la pompe et des déformations des figures d'absorption. Par ailleurs, il faut éviter des fréquences de balayage voisines de celle du réseau électrique et de ses harmoniques. Nous avons choisi une modulation de la fréquence $v^{\prime}$ de pompe à une période de $14 \mathrm{~ms}$. Le signal de modulation étant triangulaire, l'échantillonnage est effectué sur une durée de $7 \mathrm{~ms}$ correspondant au front montant ou descendant du triangle. Ceci est obtenu en déclenchant extérieurement la rampe de l'horloge de l'échantillonneur à l'aide d'impulsions fournies par le générateur de signaux triangulaires au début de chaque cycle.

La figure 2 illustre l'amélioration spectaculaire du rapport signal sur bruit obtenu. La constante d'intégration est de $20 \mathrm{~s}$. On doit noter que dans ce fonctionnement il n'y a aucun décalage en temps entre le signal

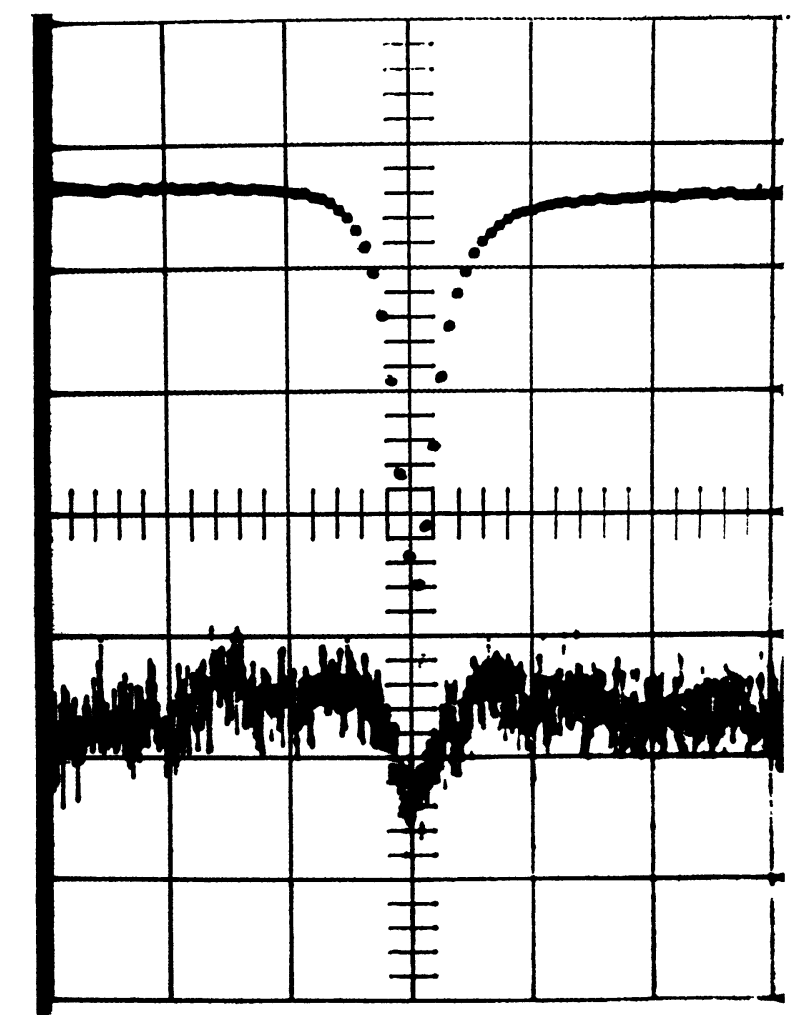

FIG. 2. - Amélioration du rapport signal sur bruit par échantillonnage. Trace inférieure : Signal avant échantillonnage. Trace supérieure : Signal après échantillonnage.

échantillonné et le signal direct et ceci quels que soient les retards au déclenchement des rampes de l'échantillonneur et de l'oscilloscope. A un instant donné, le signal issu de l'échantillonneur correspond à celui qui est introduit au même moment à l'entrée de l'appareil. Ce point est particulièrement important pour les mesures de fréquence sur des signaux échantillonnés. 
Dans les domaines usuels de puissance de sonde, les sensibilités obtenues par les techniques de démodulation synchrone et d'échantillonnage sont du même ordre de grandeur et généralement largement suffisantes. La première technique est préférable lorsque l'on désire une bonne métrologie de fréquence et elle permet seule les études en fréquence de sonde balayée. La deuxième méthode permet d'éliminer les difficultés liées à la réjection insuffisante du circulateur commutable dans les expériences à forte puissance de pompe et d'étudier rapidement les variations de forme des signaux en fonction des divers paramètres expérimentaux. Pratiquement, nous avons fréquemment effectué la mise au point des programmes expérimentaux en utilisant l'échantillonneur, les enregistrements définitifs étant obtenus en démodulation synchrone.

Dans les expériences à très basse pression, on est amené, lorsque l'on désire éviter la saturation, à employer des puissances de sonde très faibles $(10 \mu \mathrm{W}$ environ).

Dans ce cas, la sensibilité des procédés de détection directe est insuffisante et l'on est amené à les utiliser en conjonction avec une détection superhétérodyne.

Dans le cas général, ce procédé présente également l'avantage d'assurer une meilleure protection contre le signal parasite dû aux résidus de puissance de pompe arrivant sur le détecteur [15].

I. 5 Mesure des fRÉQueNCES. - Les techniques de mesure de fréquences sont tout à fait différentes selon le procédé de détection utilisé (ou de seconde détection avec une première détection superhétérodyne). Dans les deux cas, les fréquences fixes sont mesurées par simple comptage. Lorsque l'on utilise le procédé d'échantillonnage, le balayage est rapide et la mesure de fréquence s'effectue à l'aide d'un récepteur. Ce récepteur est attaqué par le signal issu de l'O. F. P. et fournit un marqueur chaque fois que la fréquence d'accord du récepteur est égale à celle de l'oscillateur. En appliquant ce marqueur sur la deuxième voie de l'oscilloscope d'observation du signal échantillonné, on peut, en ajustant la fréquence d'accord du récepteur (20 à $33 \mathrm{MHz}$ ), le placer successivement sur les différents points remarquables de la figure d'absorption et mesurer les fréquences correspondantes.

Pratiquement, en raison des niveaux importants de signaux, de la nécessité d'adapter de façon précise la bande passante à la vitesse de balayage et de pouvoir définir rapidement la fréquence d'accord, nous avons été amenés à réaliser un récepteur conçu spécialement à cet effet [11].

Il s'agit d'un récepteur superhétérodyne de fréquence intermédiaire $100 \mathrm{kHz}$ sans réjection de la fréquence image et de la bande passante continûment réglable entre 1 et $20 \mathrm{kHz}$. L'oscillateur local est suivi d'un amplificateur séparateur qui fournit le signal requis pour la mesure de sa fréquence au compteur numérique (Rochar A 1439).

Dans la technique de démodulation synchrone, la vitesse de balayage est très faible. On admet générale- ment que, dans l'observation d'une raie, la durée de passage sur la demi-largeur à mi-hauteur doit être de l'ordre de vingt à cinquante fois la constante d'intégration. En raison de la complexité plus grande des figures d'absorption observées en double résonance et pour éviter les déplacements des maximums de ces figures, nous avons habituellement réalisé des balayages d'une durée de cent secondes pour une constante de temps d'intégration de trois cents millisecondes. Dans ces conditions, on effectue bien entendu des enregistrements graphiques. Le repérage de la fréquence balayée s'effectue grâce à des marqueurs appliqués en « modulation $\mathrm{Z}$ ». Chaque marqueur se traduit par l'apparition d'un blanc sur la courbe enregistrée grâce à une courte levée de la plume de l'enregistreur. Le dispositif réalisé a fait l'objet d'une publication [16].

I. 6 SeNSibiLITÉ DU SPECTROMÈTRE. - Nous avons mesuré cette sensibilité par une technique de double modulation. La plus faible variation $\Delta A$ du coefficient d'absorption du gaz que le spectromètre puisse mettre en évidence avec un rapport signal sur bruit égal à un est de l'ordre de $1,5 \times 10^{-8} \mathrm{~cm}^{-1}$. Cette sensibilité est du même ordre que celles qui sont annoncées en spectrométrie Stark, toutes choses égales par ailleurs (vitesse de commutation, constante de temps, gamme hyperfréquence, etc...).

II. Résultats expérimentaux. - En l'absence de tout effet de saturation, la puissance absorbée par le gaz est directement proportionnelle à la puissance de sonde. En disposant d'un détecteur linéaire en puissance, on obtiendrait donc dans ce cas une information modulée proportionnelle à la variation de puissance absorbée due au pompage. Pratiquement, la sensibilité des détecteurs hyperfréquences dépend considérablement de leur polarisation et, par conséquent, en l'absence de polarisation extérieure, de la puissance hyperfréquence qu'ils reçoivent.

A l'aide d'un atténuateur, nous ramenons cette puissance à une valeur constante en fixant la tension continue détectée par le cristal.

Dans ces conditions, l'information modulée recueillie sur le détecteur est indépendante de la puissance de sonde en l'absence d'effets de saturation ; l'apparition de ces effets se traduit alors par un écrasement des figures d'absorption.

Nous avons analysé par cette technique l'influence de la puissance de sonde sur les figures d'absorption obtenues dans les études à fréquence de sonde fixe et à fréquence de pompe fixe.

II.1 ETUdes A FRÉQuenCe DE SONDE FIXE. - Ces études peuvent être entreprises indifféremment par les techniques de démodulation synchrone ou d'échantillonnage. Dans le premier cas, le spectromètre fournit un signal proportionnel à la différence des absorptions en présence et en absence de pompage ; dans le deuxième cas, on obtient la courbe représentative des variations de l'absorption en fonction de la fréquence de pompe. Les deux enregistrements ne diffèrent que par 
un décalage en ordonnée car l'absorption en absence de pompage est évidemment indépendante de la fréquence de pompe. Pratiquement, les études de saturation ne nécessitant pas de métrologie de fréquence, nous avons utilisé la technique d'échantillonnage de mise en œuvre plus rapide. Pour explorer un domaine important de variation de la puissance de sonde, il faut que, pour la plus faible puissance de sonde, le détecteur conserve une sensibilité suffisante. Cette sensibilité sera en effet maintenue constante en cours d'étude par le jeu combiné des atténuateurs placés en tête et en bout de cellule. Pratiquement, avec une tension continue détectée de $5 \mathrm{mV}$ (sur $1 \mathrm{M} \Omega$ ), on peut explorer des variations de $50 \mathrm{~dB}$ de la puissance de sonde.

Le réglage du paramètre de pompage $M^{\prime}$ (cf. : III) s'effectue par la méthode de modulation de la puissance de pompe et de démodulation synchrone. On utilise une méthode de zéro : pour $v$ égal à $v_{0}$ et $v^{\prime}$ égal à $v_{0}^{\prime}$, on sait en effet que l'absorption en présence de pompage est égale à l'absorption en absence de pompage lorsque $M^{\prime}$ est égal à 1,96; expérimentalement, ceci correspond à un signal nul à la sortie de la détection synchrone. L'atténuateur calibré $5 \mathrm{~mm}$ (Fig. 1) permet ensuite d'ajuster $M^{\prime}$ à la valeur voulue par un procédé indirect. Ce procédé consiste, par des variations de sens opposé des atténuations introduites par la grille et l'atténuateur, à maintenir constante la tension détectée par le cristal $\mathrm{X}^{\prime}$. On ajuste ainsi une puissance importante par un atténuateur calibré qui ne supporte pas cette puissance. Le réglage de $M^{\prime}$ effectué, on laisse le circulateur en position passante $(1,2,3,4)$ et on réalise l'étude en échantillonnage.

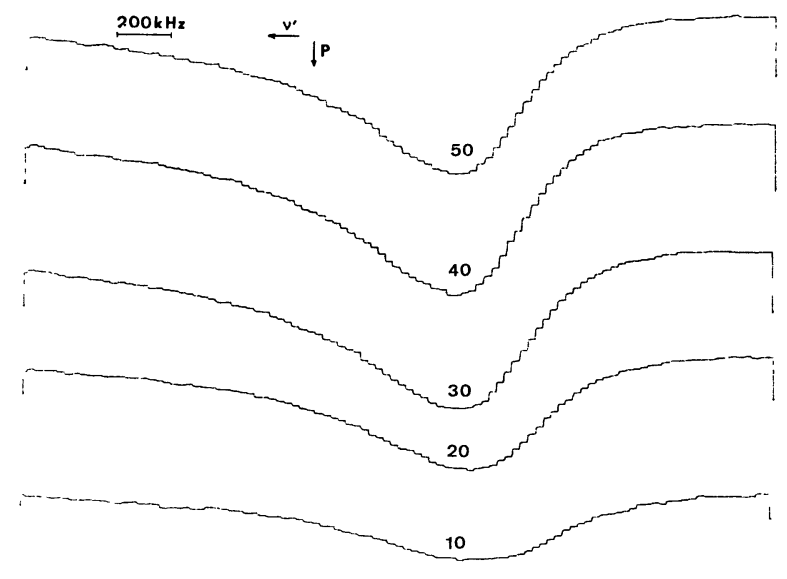

0

FIG. 3. - Phénomène de saturation d'absorption à fréquence de sonde fixe. Etude expérimentale. Chaque courbe est affectée d'un repère mentionnant l'atténuation en $\mathrm{dB}$ apportée à la puissance de sonde par rapport à une puissance de référence.

La figure 3 donne les enregistrements obtenus pour $M^{\prime}$ égal à 1,96 et une fréquence $v$ telle que :

$$
v-v_{0}=320 \mathrm{kHz} \text {. }
$$

La pression du gaz mesurée à la jauge de Mac Leod est égale à 11,8 mtorrs. La fréquence $v^{\prime}$ est balayée sur un intervalle de 2,2 MHz. Les échelons observés dans le tracé des enregistrements correspondent à l'exploration des différents canaux de l'échantillonneur. Nous avons évité de lisser ces courbes car ce procédé introduit un léger écrasement des figures d'absorption. Les enregistrements de la figure 3 et l'étude des amplitudes des maximums d'absorption en fonction de la puissance de sonde (Fig. 4) montrent que les effets de saturation n'apparaissent que pour une puissance de sonde quarante fois supérieure à la puissance minimum utilisée. Les études du même type entreprises pour des écarts $\left(v-v_{0}\right)$ plus importants montrent que la puissance de sonde correspondant au seuil de saturation est d'autant plus élevée que l'écart $\left(v-v_{0}\right)$ est plus grand.

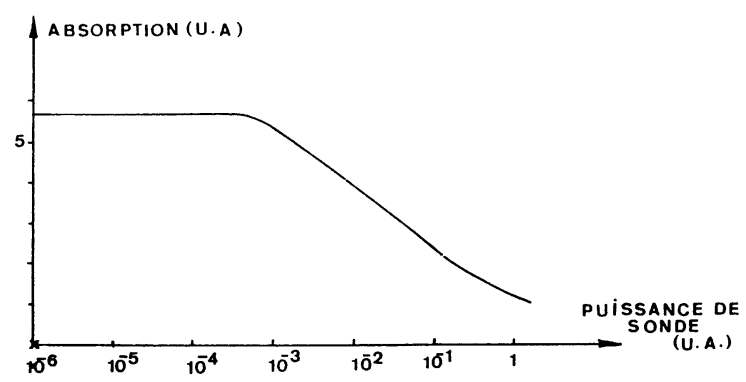

FIG. 4. - Etude des amplitudes des maximums d'absorption en fonction de la puissance de sonde. Dépouillement des enregistrements 3 .

Pour la même valeur du paramètre $M^{\prime}\left(M^{\prime}=1,96\right)$, il paraît intéressant d'étudier le cas où la fréquence de sonde est exactement égale à celle de la transition correspondante $\left(v=v_{0}\right)$.

On peut prévoir en effet, dans ce cas, l'existence d'un optimum pour la puissance de sonde. A très faible puissance de sonde, l'étude théorique de l'absorption montre que la variation d'absorption due au pompage est nulle quelle que soit la fréquence de pompe. Il en est de même à forte puissance de sonde en raison des phénomènes de saturation. Entre ces deux valeurs de la puissance de sonde, il existe donc un cas intermédiaire correspondant à un maximum d'absorption relative. La figure 5 donne l'aspect des résultats expérimentaux. On observe effectivement un optimum qui est obtenu pour une puissance relative de sonde de $26 \mathrm{~dB}$ environ. L'aspect des enregistrements n'est cependant pas exactement conforme à ce qui était prévu. En particulier, la courbe obtenue à très faible puissance de sonde présente un maximum très plat affecté d'un creusement au centre alors que l'on attend une absorption constante. Ceci peut être attribué aux inhomogénéités de puissance de pompe dans la cellule, et, éventuellement à des levées de dégénérescences $m$ par le champ de pompe. Nous reviendrons sur ces points importants à la fin de notre étude. Qualitativement, on peut cependant dire que l'effet observé serait la superposition d'effets correspondant à différentes valeurs du paramètre de pompage $M^{\prime}$. Les valeurs inférieures à 1,96 seraient responsables du creusement central, et les valeurs supérieures expliqueraient la forme observée loin de la fréquence $v_{0}^{\prime}$. 


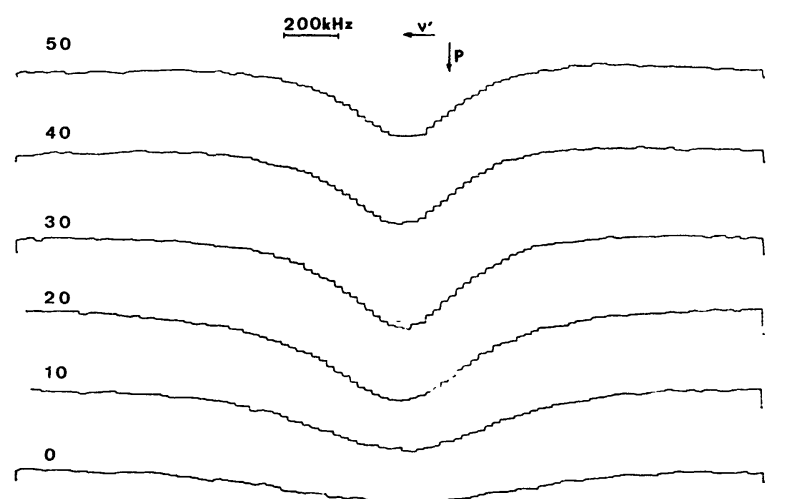

Fig. 5. - Saturation d'absorption à la résonance de sonde. Etude expérimentale.

On évite les difficultés en adoptant une valeur « moyenne » $\bar{M}^{\prime}$ notablement inférieure à 1,96 . (Nous désignons par valeur moyenne celle qui est réalisée par la technique expérimentale exposée précédemment.)

L'étude expérimentale dans le cas où $\bar{M}^{\prime}$ est égal à 0,2 montre que la saturation de sonde apparaît pour des puissances de sonde plus faibles que celles observées dans l'étude de la figure 4. De manière générale, il semble donc que les phénomènes de saturation soient d'autant plus importants que la fréquence $v$ de sonde est voisine de $v_{0}$. Il reste à confirmer ce résultat par des études à fréquence de pompe fixe.

II. 2 ETUde A FRÉQUeNCE DE POMPE FIXE. - Cette étude ne peut être entreprise que par la technique de modulation de la puissance de pompe et de démodulation synchrone du signal détecté à la fréquence de sonde. En balayant la fréquence de sonde, la raie de sonde se soustrait de la figure d'absorption en présence de pompage pour donner la figure effectivement observée. Lorsque la fréquence de pompe est assez éloignée de $v_{0}^{\prime}$, cette soustraction affecte essentiellement

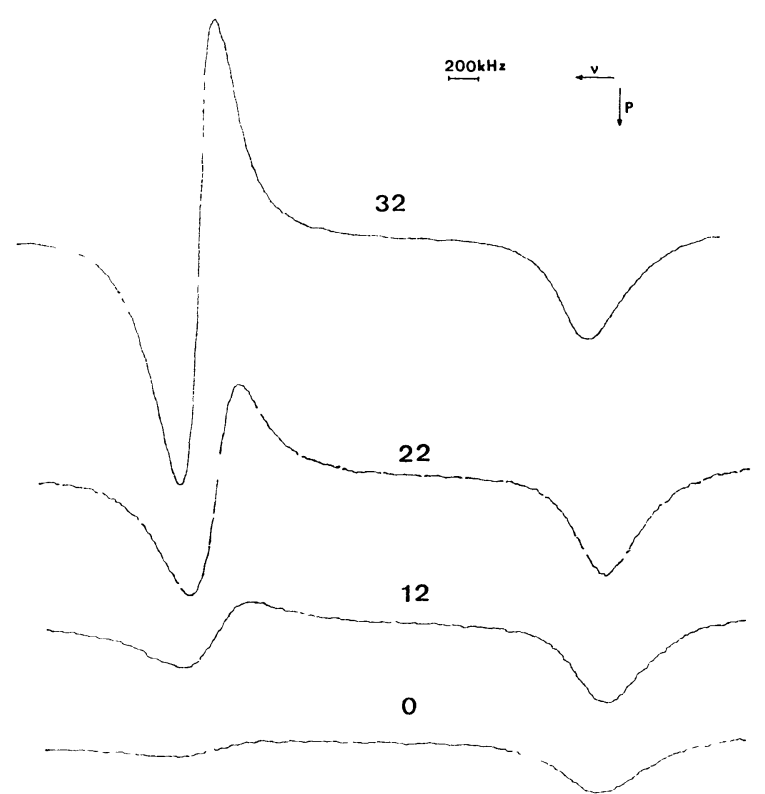

Fig. 6. - Saturation de la résonance centrale et de l'extra-pic. Etude expérimentale. la résonance centrale et fournit au voisinage de $v_{0}$ une courbe qui a grossièrement l'aspect d'une raie différenciée (D). La saturation de sonde affecte évidemment à la fois la résonance centrale et la raie obtenue en absence de pompage. On peut estimer qualitativement l'importance relative de ces deux effets en examinant la largeur et l'amplitude de deux composantes de (D). La figure 6 reproduit les enregistrements obtenus dans les conditions suivantes:

$$
p=7,2 \text { mtorrs } ; \quad v^{\prime}-v_{0}^{\prime}=3 \mathrm{MHz} ; \quad M^{\prime}=5 .
$$

La fréquence de sonde est balayée sur un intervalle de $4 \mathrm{MHz}$ environ. En raison de la faible amplitude des signaux obtenus dans ces conditions, il a été nécessaire d'utiliser des puissances de sonde plus importantes pour conserver un bon rapport signal sur bruit. Chaque courbe du réseau 6 est affectée d'un repère mentionnant la puissance relative de sonde dans l'échelle utilisée précédemment (II.1). La saturation de sonde sur l'extra-pic [2] n'apparaît qu'à partir d'une puissance relative de $16 \mathrm{~dB}$ environ; au contraire, la saturation de la résonance centrale apparaît dès les plus faibles puissances de sonde et, en appliquant la règle qualitative indiquée ci-dessus, on peut remarquer que la résonance centrale et la raie de sonde observée en l'absence de pompage sont sensiblement affectées de la même manière par la saturation. On peut noter également l'effet de la saturation sur la largeur des deux composantes de l'absorption. L'élargissement le plus important affecte la résonance centrale ; celui de l'extra-pic est plus faible et, de plus, il paraît masqué par d'autres phénomènes (inhomogénéité de $M^{\prime}$ notamment).

Une étude plus fine montre également un léger déplacement de l'extra-pic, dû à la puissance de sonde, vers la résonance centrale. En adoptant comme origine la fréquence de l'extra-pic aux puissances de sonde les plus faibles, le dépouillement des enregistrements effectués permet de dresser le tableau suivant :

$\begin{array}{lllllll}\begin{array}{c}\text { Puissance de } \\ \text { sonde rela- }\end{array} & 42 & 32 & 22 & 12 & 6 & 0 \\ \begin{array}{c}\text { tive (dB) } \\ \Delta v(\mathrm{kHz})\end{array} & 0 & 5 & 15 & 35 & 55 & 75\end{array}$

Comparés à la largeur de l'extra-pic (de l'ordre de $400 \mathrm{kHz}$ ), ces déplacements sont faibles et négligeables dans le cas des puissances de sonde habituellement utilisées.

II. 3 CoNCLUSION. - On peut résumer les résultats obtenus dans les études de saturation de sonde en indiquant de manière générale que la saturation de sonde affecte :

- l'amplitude des figures d'absorption mais d'autant moins que la fréquence de sonde est plus éloignée de la fréquence de la transition;

- la largeur des figures d'absorption à fréquence de sonde balayée. 
En revanche, les fréquences des maximums d'absorption ne paraissent guère modifiées en première approximation.

III. Interprétation théorique. - L'interprétation des résultats expérimentaux nécessite un calcul de l'absorption du gaz sans hypothèse particulière sur l'ordre de grandeur des paramètres $M^{\prime}$ et $M$ caractéristiques des puissances de pompe et de sonde. Le formalisme des théories de perturbation dépendant du temps [17], [18] qui nécessitent pratiquement un calcul de l'opérateur d'évolution dans la description d'interaction [2] est mal adapté à un tel propos. Nous avons donc abandonné ce formalisme pour utiliser une méthode basée sur l'utilisation d'opérateurs « habillés » [19].

Le principe de cette méthode est d'inclure les champs hyperfréquences dans le système étudié, le problème à résoudre est alors un calcul de perturbations stationnaires dans un cas quasiment dégénéré et on évite par ce biais l'essentiel des difficultés rencontrées dans les calculs de perturbation dépendant du temps.

Plus précisément, le formalisme que nous avons développé [9] est dérivé de celui exposé par Di Giacomo et Santucci dans un article récent [8]. Nous nous contenterons ici d'exploiter numériquement les résultats théoriques que nous avons obtenus dans des conditions qui correspondent sensiblement aux conditions expérimentales. Nous distinguerons donc également les études à fréquence de sonde fixe et à fréquence de pompe fixe. Nous ajouterons un paragraphe important relatif aux effets dus à la levée de dégénérescence $m$ qui permet d'expliquer certaines divergences. Remarquons que les calculs théoriques sont effectués en introduisant des grandeurs réduites.

- Fréquences réduites :

$$
z=2 \pi\left(v-v_{0}\right) \tau ; \quad z^{\prime}=2 \pi\left(v-v_{0}\right) \tau
$$

où $\tau$ est le temps de libre parcours moyen des molécules.

Ceci revient à exprimer les écarts $v-v_{0}$ et $v^{\prime}-v_{0}^{\prime}$ en adoptant comme unité la demi-largeur à mi-hauteur des raies de sonde et de pompe non saturées.

- Puissances réduites :

$$
M=\left|\frac{\mu_{23} E \tau}{2 \hbar}\right|^{2} ; \quad M^{\prime}=\left|\frac{\mu_{12} E^{\prime} \tau}{2 \hbar}\right|^{2}
$$

où $\mu_{23}$ et $\mu_{12}$ sont les éléments de matrice dipolaire électrique relatifs aux transitions de sonde et de pompe. $E$ et $E^{\prime}$ désignent les amplitudes des champs électriques de sonde et de pompe.

\section{- Absorption réduite.}

L'unité adoptée est la puissance absorbée en l'absence de pompage, à la résonance de sonde et en négligeant les effets de saturation.

III.1 CAlCul A FRÉQueNCE DE SONDE FIXE. — La figure 7 donne les résultats d'une exploitation numé- rique effectuée sur calculateur Bull M 40 dans le cas où :

$$
z=2 ; M^{\prime}=1,96 ; M=0,01-0,1-1-10 .
$$

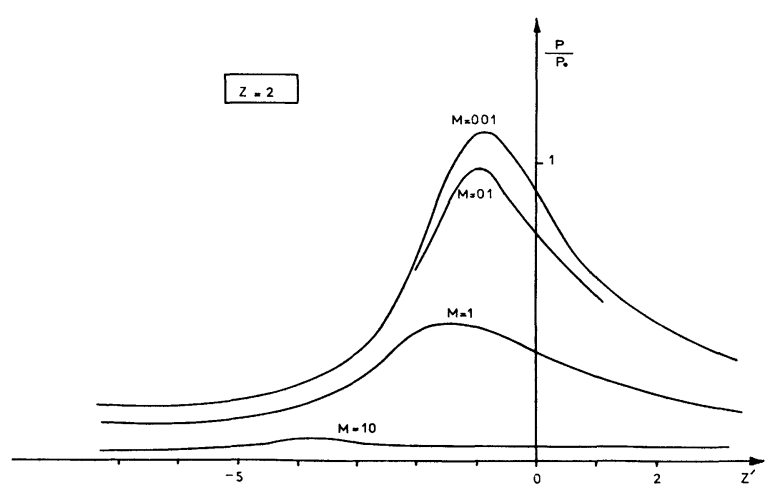

FIG. 7. - Etude théorique de la saturation d'absorption à fréquence de sonde fixe.

Ce sont pratiquement les conditions des enregistrements de la figure 3. On observe que la saturation de sonde se manifeste pour une valeur du paramètre $M$ assez faible $(0,1)$. Une saturation équivalente $(10 \%)$ est obtenue expérimentalement avec une puissance relative de sonde de $30 \mathrm{~dB}$.

Le recoupement des résultats théoriques et expérimentaux est assez bon. On note cependant que la saturation observée expérimentalement est plus progressive que celle qui est prévue par le calcul. Nous retrouverons ce phénomène dans toutes les études de saturation (absorption et dispersion). On peut l'attribuer à l'inhomogénéité de la puissance de sonde (atténuation et ondes stationnaires) et à une surestimation de cette puissance lorsque l'oscillateur de sonde est fortement couplé à la cellule (courbes expérimentales 0 et $10 \mathrm{~dB})$.

Dans les mêmes conditions que précédemment mais lorsque la sonde est résonnante $(z=0)$, on obtient le réseau de la figure 8 . Comme on peut le prévoir, on observe un écrasement de la figure d'absorption à très faible puissance de sonde $(M \sim 0)$ conformément aux études antérieures et à très forte puissance de sonde en raison des phénomènes de saturation. Comme nous

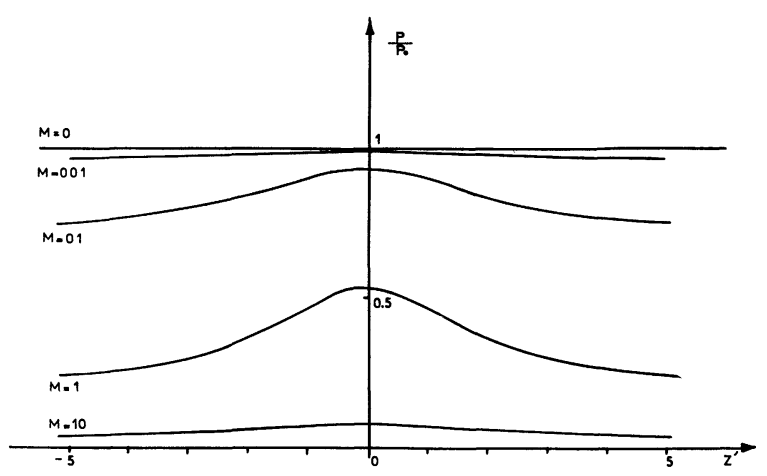

Fig. 8. - Etude théorique de la saturation d'absorption à la résonance de sonde. 
l'avons observé expérimentalement (Fig. 5), il existe une puissance de sonde pour laquelle l'amplitude de la figure d'absorption est maximum. Nous convenons de désigner ainsi la différence des absorptions au centre $\left(z^{\prime}=0\right)$ et très loin du centre $\left(z^{\prime} \rightarrow \infty\right)$ seule accessible expérimentalement. Nous n'avons pas recherché à préciser la valeur optimum du paramètre $M$ car les enregistrements expérimentaux (40 et 50 de la Fig. 5) obtenus pour les faibles valeurs de $M$ diffèrent considérablement des courbes théoriques $(0$ et 0,01$)$. On interprète convenablement ces divergences en tenant compte de l'inhomogénéité de la puissance de pompe dans la cellule et des effets liés à la dégénérescence $m$.

III. 2 CAlCUl A FRÉQUENCE DE POMPE FIXE. - Dans notre technique expérimentale, la raie de sonde se soustrait de l'absorption en présence de pompage pour fournir les enregistrements obtenus. Il est nécessaire d'effectuer la même opération dans notre traitement théorique pour permettre une confrontation commode. Compte tenu des phénomènes de saturation [18], la puissance absorbée en absence de pompage (raie de sonde) est de la forme $1 /\left(1+z^{2}+4 M\right)$ en adoptant les unités réduites. Il suffit donc de retrancher

$$
\frac{1}{1+z^{2}+4 M}
$$

de la grandeur calculée.
La figure 9 illustre le résultat d'un calcul de ce type entrepris dans le cas suivant :

$$
M^{\prime}=5 ; z^{\prime}=20 ; M=0-0,1-10-100 .
$$

Ceci correspond très approximativement aux conditions d'expérience des enregistrements 6 . On vérifie bien l'essentiel des résultats expérimentaux :

- Saturation de la résonance centrale pour des puissances de sonde beaucoup plus faibles que celles qui sont nécessaires pour réaliser la saturation de l'extra-pic.

- Saturation identique de la raie de sonde et de la résonance centrale en présence de pompage.

- Elargissement de la résonance centrale beaucoup plus important que celui qui est observé sur l'extra-pic.

- Léger déplacement de l'extra-pic vers la résonance centrale lorsque l'on augmente le paramètre de saturation de sonde.

De façon plus quantitative, on peut rapprocher l'enregistrement obtenu pour une puissance relative de sonde de $32 \mathrm{~dB}$ de la courbe calculée pour $M$ égal à 0,1 . Il y aurait déjà pour cette puissance de sonde un effet de saturation sur la résonance centrale. Comme précédemment, la saturation observée expérimentalement est plus progressive que celle qui est prévue théoriquement. Le fait que ce phénomène affecte plus

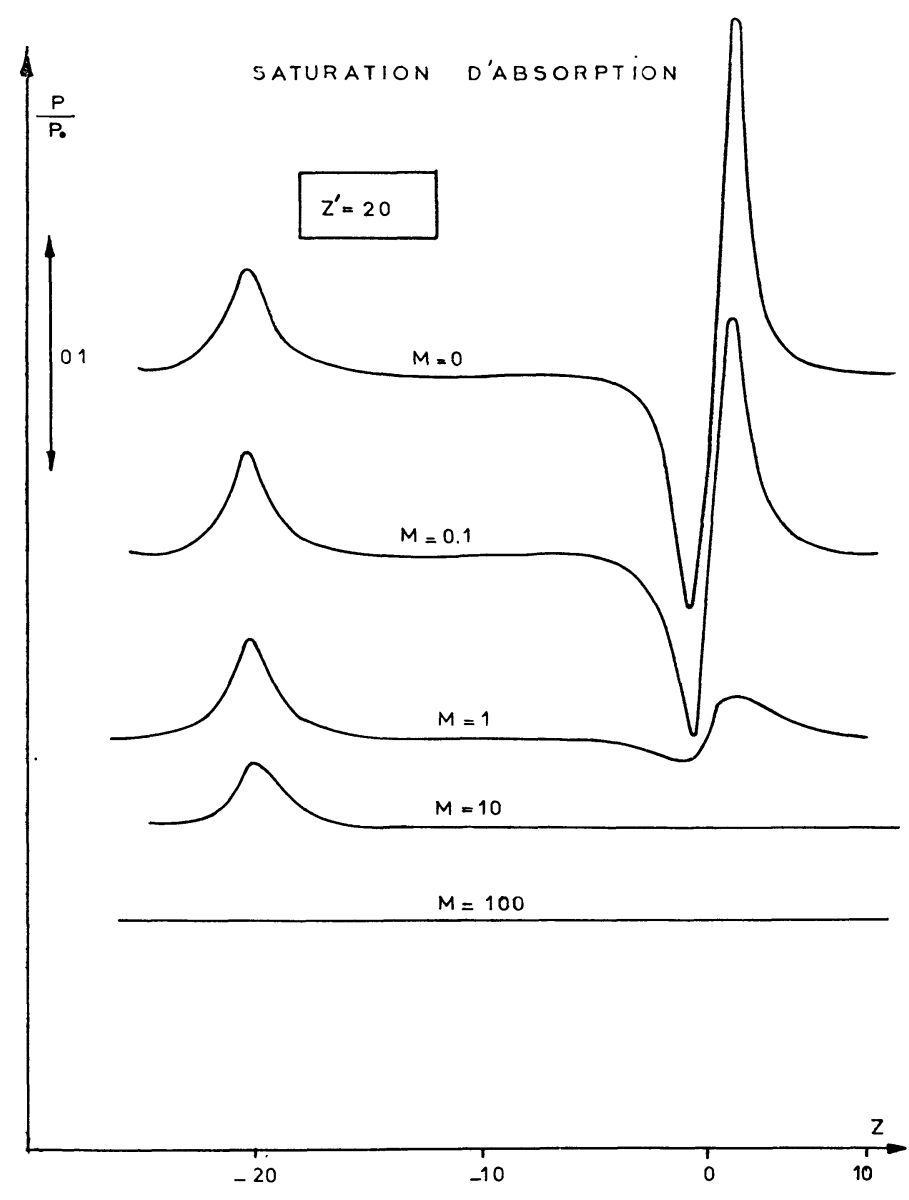

FIG. 9. - Etude théorique de la saturation de l'extra-pic et de la résonance centrale $\left(M^{\prime}=5, z^{\prime}=20\right)$. 
sensiblement la résonance centrale que l'extra-pic rend plausible les hypothèses avancées et en particulier celle d'une surestimation des fortes puissances de sonde.

III. 3 EFFETS DUS A LA DÉGÉNÉRESCENCE $m$. - Les calculs que nous avons effectués aussi bien dans le formalisme de l'opérateur d'évolution que dans celui de la molécule habillée supposent les niveaux d'énergie de la molécule isolée non dégénérés. Nous nous proposons de prendre en compte la dégénérescence $m$ qui est levée par les champs électriques appliqués [1].

De manière générale, l'opérateur composante du moment cinétique $\mathbf{J}$ suivant une direction fixe de l'espace commute avec l'hamiltonien de rayonnement $H_{\mathrm{R}}$ et avec l'hamiltonien de la molécule isolée $H_{\mathrm{S}}$ mais ne commute pas avec l'hamiltonien total $H$. Cependant, dans le cas particulier où les champs électriques de pompe et de sonde ont même direction notée $z^{\prime} z$, on montre facilement que l'opérateur $J_{z}$ commute avec le terme d'interaction $H_{\mathrm{I}}$ et donc également avec $H$. Si on se place dans la base des kets propres communs à $H_{\mathrm{R}}, H_{\mathrm{S}}, J^{2}$ et $J_{z}$, le problème se sépare entièrement par rapport au nombre quantique $m$ et la puissance absorbée peut s'écrire :

$$
P=\sum_{m} P\left[M(m), M^{\prime}(m)\right]
$$

où $M(m)$ et $M^{\prime}(m)$ sont les paramètres de saturation et de pompage associés aux éléments de matrice

$$
<2, m|\hat{\mu}| 3, m>\text { et }\langle 1, m|\hat{\mu}| 2, m>
$$

du moment dipolaire et où $P$ est l'expression de la puissance absorbée obtenue dans un calcul négligeant la dégénérescence $m$. Remarquons que l'effet de la dégénérescence se manifeste à la fois sur le paramètre de pompage $M^{\prime}$ et sur le paramètre de saturation $M$. Ce deuxième effet souvent négligé est important même en l'absence de phénomène de saturation car il pondère les composantes de l'absorption associées aux différentes valeurs $M^{\prime}(m)$ du paramètre de pompage.

Appliquons les résultats précédents à notre cas particulier. Les états propres $|1\rangle,|2\rangle,|3\rangle$ de la molécule isolée correspondent à des nombres quantiques $J$ égaux à 6,6 et 5 .

Le paramètre de pompage $M^{\prime}(0)$ est nul. La contribution correspondante à l'absorption est la même en présence et en absence de pompage et n'apparaît pas expérimentalement en raison des techniques employées.

Compte tenu de cette remarque, la puissance absorbée s'écrit :

$$
P=\sum_{m=1}^{5} P\left[M(m), M^{\prime}(m)\right] .
$$

Pour obtenir les valeurs absolues des paramètres $M(m)$ et $M^{\prime}(m)$, il est nécessaire de connaître les éléments de matrice réduits de $\hat{\mu}$. On les déduit facilement des tableaux d'intensité de raie et des données spectro- scopiques [4] de $\mathrm{SO}_{2}\left(\mu_{\mathrm{a}}=\mu_{\mathrm{c}}=0 ; \mu_{\mathrm{b}}=1,59\right.$ Debye $=$ $=5,3 \times 10^{-30}$ M. K. S. A. ; $\left.\kappa=-0,94\right)$. Pratiquement, il est plus avantageux d'exprimer $M(m)$ et $M^{\prime}(m)$ à partir des paramètres $M$ et $M^{\prime}$ considérés dans les calculs précédents et qui sont obtenus par addition des contributions $M(m)$ et $M^{\prime}(m)$ correspondant aux diverses valeurs de $m$. On obtient immédiatement :

$$
M(m)=\frac{M}{286}\left(36-m^{2}\right) ; \quad M^{\prime}(m)=\frac{M^{\prime}}{110} m^{2} .
$$

Il est évident que la dégénérescence $m$ réduit fortement l'efficacité du pompage. En particulier, on peut préciser la valeur de $M^{\prime}$ correspondant à l'égalité des absorptions en présence et en absence de pompage à la résonance de sonde et de pompe. Elle est définie par :

$$
\sum_{m=1}^{5} P\left[M(m), M^{\prime}(m)\right]=\sum_{m=1}^{5} P[M(m), 0] .
$$

En négligeant la saturation de sonde, et en introduisant le paramètre $\rho$ égal $\left(v_{0}+v_{0}^{\prime}\right) / v_{0}$, on obtient :

$$
\begin{aligned}
\frac{M^{\prime}}{110} \sum_{m=1}^{5} m^{4}\left(36-m^{2}\right) & =\left(\frac{3}{4} \rho-1\right) \sum_{m=1}^{5} m^{2}\left(36-m^{2}\right) \\
M^{\prime} & \simeq 15 .
\end{aligned}
$$

Rappelons qu'en négligeant la dégénérescence $m$ ce résultat est obtenu avec un paramètre de pompage $M^{\prime}$ égal à 1,96 . Notons que la valeur $M^{\prime}=15$ est plus en rapport avec les estimations directes que l'on peut faire du paramètre $M^{\prime}$ en évaluant le champ électrique de pompe dans la cellule. Il en résulte que nous rencontrerons pour certaines composantes de l'absorption des valeurs de paramètre de pompage $M^{\prime}(m)$ beaucoup plus grandes que celles rencontrées dans les calculs précédents.

Remarquons que lorsque la saturation de sonde est négligeable, l'amplitude relative des différentes composantes $m$ de l'absorption est proportionnelle au paramètre $M(m)$ qui prend sa plus grande valeur pour les composantes les plus faiblement pompées.

$$
\begin{array}{cccccc}
\begin{array}{ccc}
m \\
\text { - }
\end{array} & \pm 1 & \pm 2 & \pm 3 & \pm 4 & \pm 5 \\
\begin{array}{c}
\text { Valeur relative } \\
\text { de } M^{\prime}(m)
\end{array} & 0,04 & 0,16 & 0,36 & 0,64 & 1 \\
\begin{array}{c}
\text { Valeur relative } \\
\text { de } M(m)
\end{array} & 1 & 0,91 & 0,72 & 0,57 & 0,31
\end{array}
$$

Dans le cas de faibles pompages, on n'observe pas de différence entre les résultats obtenus en tenant compte de la dégénérescence $m$ et en la négligeant.

En revanche, dans le cas des forts pompages (Fig. 10 ; $z=40 ; M^{\prime}(m)=30 \mathrm{~m}^{2}$ ), la dégénérescence est levée par le champ de pompe et on distingue cinq composantes dans la figure d'absorption. Ces composantes classées par ordre de fréquence de pompe croissante correspondent à $m$ égal respectivement à $\pm 1\left(z^{\prime} \simeq-39\right), \pm 2\left(z^{\prime} \simeq-37\right), \pm 3\left(z^{\prime} \simeq-33\right)$, $\pm 4\left(z^{\prime} \simeq-28\right)$ et $\pm 5\left(z^{\prime} \simeq-22\right)$. Il faut remarquer 


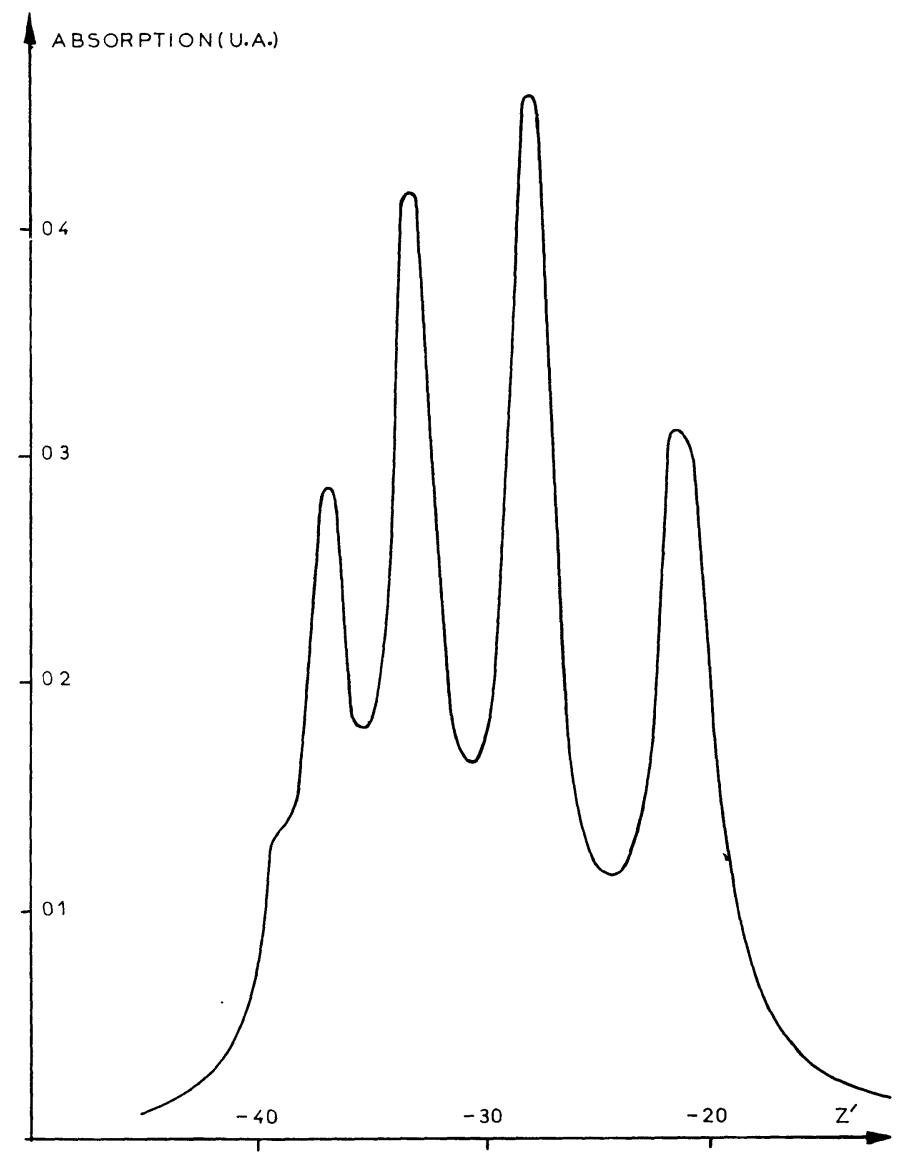

Fig. 10. - Levée de la dégénérescence $m$. Etude théorique dans le cas des forts pompages.

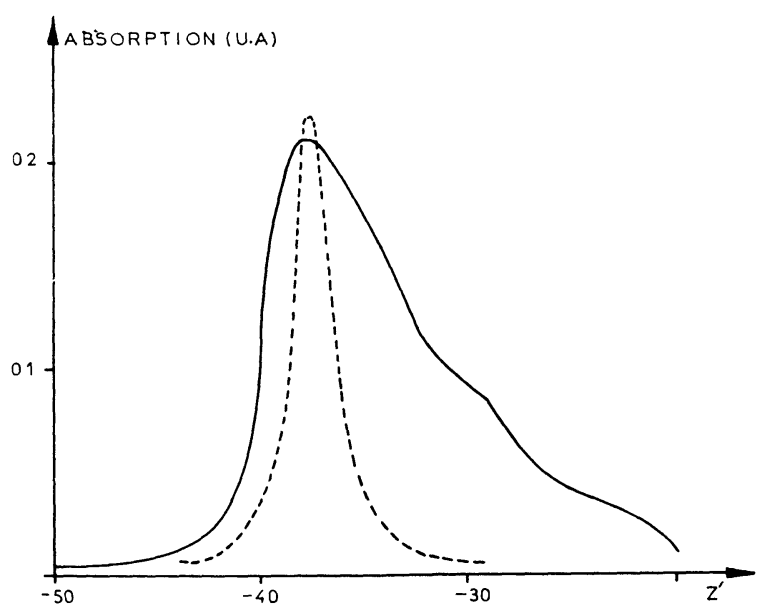

FIG. 11. - Effets combinés de la dégénérescence $m$ et de l'inhomogénéité de la puissance de pompe. En pointillé : courbe obtenue en négligeant ces deux effets.

qu'une telle résolution est difficile à mettre en évidence expérimentalement [20]. Elle est généralement masquée par les effets liés à l'inhomogénéité du champ de pompe. Ce phénomène est analogue à celui observé en spectrométrie Stark où l'inhomogénéité du champ électrique se traduit par une perte sensible de résolution. Pratiquement, l'homogénéité d'un champ hyperfréquence est beaucoup plus difficile à maîtriser que celle d'un champ

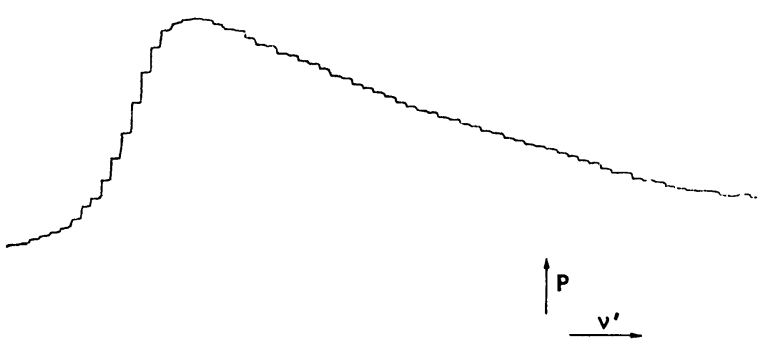

Fig. 12. - Mise en évidence expérimentale des effets de la dégénérescence $m$ et de l'inhomogénéité du pompage.

statique. Dans notre cellule, si le paramètre de pompage $M^{\prime}(m)$ de la composante $m$ de l'absorption est égal à $30 \mathrm{~m}^{2}$ à l'entrée, il n'est que de $3 \mathrm{~m}^{2}$ à l'extrémité du guide. On observe donc un mélange des composantes associées à des valeurs différentes de $m$. Un calcul effectué dans ces conditions et tenant compte à la fois de la dégénérescence $m$ et de l'inhomogénéité du champ de pompe donne des résultats conformes à ce que nous attendons (Fig. 11). On note en effet un élargissement et une dissymétrie importante de la figure d'absorption; la courbe tracée en pointillé et obtenue en négligeant dégénérescence et atténuation, fournit un bon élément de comparaison.

Expérimentalement, nous avons effectivement obtenu des figures d'absorption de ce type. L'enregis- 
trement de la figure 12 obtenu par échantillonnage correspond sensiblement aux conditions du calcul précédent et reproduit convenablement la prévision théorique. Seuls les effets de dégénérescence $m$ permettent d'interpréter de tels résultats.

Indiquons également qu'en effectuant un calcul analogue des figures d'absorption obtenues à la résonance (cf. II.1, Fig. 5) on obtient une correspondance parfaite entre le résultat théorique et le résultat expérimental précédemment inexpliqué.

Conclusion. - Les phénomènes de saturation observés en double résonance diffèrent assez notablement de ceux que l'on obtient dans le cas d'une raie élargie par saturation. Il existe en particulier des circonstances expérimentales $(z \gg 1)$ où l'on n'observe pas de saturation en double résonance alors qu'il y a saturation sensible de la raie en absence de pompage $(M>1)$. Inversement, dans d'autres cas $(z \sim 0)$, les effets de saturation sont de même type, et ceci quelles que soient les puissances relatives de pompe et de sonde. La condition de non-saturation habituellement avancée en double résonance $\left(M \ll M^{\prime}\right)$ est donc inexacte puisqu'il existe des cas extrêmes où il n'y a pas saturation lorsque la règle $M \ll M^{\prime}$ est violée et d'autres où il y a saturation alors que cette règle est respectée.

Enfin, les calculs d'effets liés à la dégénérescence $m$ montrent que la possibilité de résoudre le problème en séparant les contributions des diverses valeurs du nombre quantique magnétique n'existe que lorsque les champs de pompe et de sonde sont à polarisations rectilignes parallèles. Cette particularité disparaît en champs croisés.

D'ores et déjà, nous avons réalisé la propagation de champs croisés dans un guide d'onde et l'étude des phénomènes de corrélation angulaire attendus devrait présenter un grand intérêt expérimental et théorique.

\section{Bibliographie}

[1] FlynN (G. W.), J. Mol. Spectr., 1968, 28, 1. Cet article contient une bibliographie assez complète des études de double résonance en spectrométrie hertzienne des gaz à basse pression.

[2] Macke (B.), Messelyn (J.) et Wertheimer (R.), $J$. Physique, 1969, 30, 665.

[3] Legrand (J.), Macke (B.), Messelyn (J.) et WertheiMER (R.), Rev. Phys. Appl., 1968, 3, 237.

[4] Bellet (J.), Thèse, Ann. Phys., 1965, 10, 827.

[5] Glorieux (P.), Legrand (J.), Macke (B.) et Messelyn (J.), Comptes rendus, 1969, 268B, 1253.

[6] MaCKe (B.) et Messelyn (J.), Comptes rendus, 1969, 269B, 712.

[7] MaCKe (B.) et Messelyn (J.), Comptes rendus, 1969, 269B, 756

[8] Di Giacomo (A.) et Santucci (S.), Nuovo Cimento, 1969, 63B, 407.

[9] GlorieuX (P.), Macke (B.) et Messei.yn (J.), Comptes rendus, 1970, 270B, 692.
[10] Geschwind (S.), Ann. New York Acad. Sci., 1952, 55, 743.

[11] Lapauw (J. M.), Mémoire d'Ingénieur C. N. A. M., Lille, 1969.

[12] Aubrun (J.), Onde Electrique, 1965, 462, 1062.

[13] Battaglia (A.), Nuovo Cimento, 1967, 49B, 145.

[14] Deldalle (A.), Comptes rendus, 1969, 269B, 979.

[15] GlorieuX (P.), Legrand (J.), MACKe (B.) et MesSelyn (J.), à paraître.

[16] Lapauw (J. M.) et Macke (B.), Rev. Phys. Appl., 1970, $5,351$.

[17] Autler (G. H.) et Townes (C. H.), Phys. Rev., 1955, $100,703$.

[18] Javan (A.), Phys. Rev., 1957, 107, 1579.

[19] Cohen-Tannoudi (C.) et Haroche (S.), Comptes rendus, 1966, 262B, 37 ; J. Physique, 1969, 30, 125 et 153.

[20] Ока (T.), Canad. Journ. Phys., 1969, 47, 2343. 